\title{
CD204-positive macrophages accumulate in breast cancer tumors with high levels of infiltrating lymphocytes and programmed death ligand-1 expression
}

\author{
MAYUKO NAGANO $^{1}$, KANAKO SAITO ${ }^{2}$, YUJI KOZUKA ${ }^{3}$, MASAKO ICHISHI ${ }^{3}$, HIROTO YUASA ${ }^{3}$, \\ AYA NORO $^{1}$, NAO IMAI ${ }^{1}$, MAI SHIBUSAWA ${ }^{1}$, MAO KIMOTO ${ }^{1}$, MAKOTO ISHITOBI $^{1}$, YASUTAKA TONO $^{2}$, \\ HIROYASU ODA ${ }^{2}$, MIKIYA ISHIHARA ${ }^{2}$, TOSHIRO MIZUNO ${ }^{2}$, TOMOKO OGAWA ${ }^{1}$ and NAOYUKI KATAYAMA ${ }^{2}$ \\ Departments of ${ }^{1}$ Breast Surgery, and ${ }^{2}$ Hematology and Oncology, Mie University Graduate School of Medicine; \\ ${ }^{3}$ Department of Pathology, Mie University Hospital, Mie 514-8507, Japan
}

Received March 22, 2020; Accepted October 6, 2020

DOI: $10.3892 / \mathrm{ol} .2020 .12297$

\begin{abstract}
Although immunotherapy has been demonstrated to be promising in triple-negative (TN) breast cancer (BC), most $\mathrm{BC}$ cases are classified as non-TN. To enrich the responders for immunotherapy regardless of their subtypes, classification based on tumor-infiltrating lymphocyte (TIL) levels and programmed death ligand-1 (PD-L1) status may be useful. However, this classification has not been fully applied to BC. Furthermore, suppressive subsets in the local tumor microenvironment, such as tumor-associated macrophages (TAMs), which promote tumor progression, cannot be ignored to overcome immunotherapy resistance. The aims of the present study were to classify primary BC cases based on the TIL levels and PD-L1 status, and to identify suppressive immune subsets in each categorized group. A retrospective analysis of 73 patients with invasive BC was performed. The frequency of TILs was evaluated in HE-stained slides (10\% cutoff), and PD-L1 levels (SP142; 1\% cutoff), as well as immune subsets $\left(\mathrm{CD}^{+}, \mathrm{CD}^{+}, \mathrm{FOXP}^{+}, \mathrm{CD} 20^{+}, \mathrm{CD}^{2} 8^{+}\right.$and $\mathrm{CD} 204^{+}$ cells) were assessed using immunohistochemistry. It was revealed that $22 \%(16 / 73)$ of the tumors were categorized as $\mathrm{TIL}^{+} \mathrm{PD}-\mathrm{L1}^{+}$, of which $69 \%(11 / 16)$ were TN type. By contrast, $66 \%(48 / 73)$ of the tumors were categorized as TIL-PD-L1 ${ }^{-}$, of which $77 \%$ (37/48) were $\mathrm{HR}^{+}$and HER2- types. The number of CD204+ M2-type macrophages was significantly associated with high histological grade $(\mathrm{P}=0.0246)$ and high Ki-67 $(\mathrm{P}=0.0152)$, whereas $\mathrm{CD} 68^{+}$macrophages were not associated with these factors. Furthermore, CD $204^{+}$macrophages and
\end{abstract}

Correspondence to: Dr Kanako Saito, Department of Hematology and Oncology, Mie University Graduate School of Medicine, 2-174, Edobashi, Tsu, Mie 514-8507, Japan

E-mail:kana-s@clin.medic.mie-u.ac.jp

Key words: breast cancer, tumor-infiltrating lymphocytes, programmed death ligand-1, tumor-associated macrophages, CD204+ macrophages
FOXP3 $^{+}$Tregs accumulated in $88 \%(14 / 16)$ and $63 \%(10 / 16)$ of $\mathrm{TIL}^{+} \mathrm{PD}-\mathrm{L}^{+}{ }^{+}$tumors, respectively, compared with $20.8 \%$ (10/48) and $27.1 \%$ (13/48) of TIL-PD-L1' tumors. In conclusion, $22 \%$ of $\mathrm{BC}$ tumors were classified as $\mathrm{TIL}^{+} \mathrm{PD}-\mathrm{Ll}^{+}(69 \%$ were $\mathrm{TN}$ ), which were enriched with suppressive immune subsets. These cell types may serve as potential novel immunotherapeutic targets.

\section{Introduction}

Until several years ago, immunotherapy in the breast cancer (BC) field was considered as the treatment with anti-HER2 agents such as trastuzumab in combination with conventional chemotherapy. Although trastuzumab has dramatically changed the treatment for HER2-type BCs, non-HER2 BCs currently have no immune-mediated therapies.

Recently, inhibition of interactions between programmed death 1 (PD-1) on activated T cells and PD-Ligand 1 (PD-L1) on antigen-presenting cells, including tumor cells (TCs), using anti-PD-1 or anti-PD-L1 antibodies has been shown to induce robust and durable responses in several cancer types including melanoma, bladder cancer, and non-small cell lung cancer (NSCLC) (1-3). BC has been generally considered low immunogenic due to low mutational load, unlike melanoma and NSCLC that harbor high mutational loads (4). Nevertheless, tumor-infiltrating lymphocytes (TILs) are often present around and/or within breast tumors and are a prognostic factor in early BC based on large prospective clinical trials of adjuvant therapies (5-7). Considering that TILs represent the presence of immune responses against tumors, immune checkpoint inhibitors (ICIs) for BC seemed to be a novel strategy.

In $\mathrm{BC}$, treatments using ICIs monotherapy or in combination with chemotherapy have been primarily investigated for triple-negative (TN) BCs owing to higher immunogenicity derived from genomic instability and a higher mutational load of TNBCs than other subtypes $(8,9)$. Interestingly, although monotherapy of atezolizumab (anti-PD-L1 antibody, Roche) for metastatic TNBC patients showed low response rate, overall survival (OS) of patients with PD- $\mathrm{L1}^{+}$tumors showed durable responses (2-year OS 25\% and 3-year OS 21\%), which is a 
great benefit of ICIs (9). Recently, a phase III trial reported that co-treatment with atezolizumab and nab-paclitaxel improved progression-free survival and overall survival in metastatic TN patients with PD- $1^{+}$tumors as compared to nab-paclitaxel treatment alone (10), indicating that immunotherapy modulating the $\mathrm{PD}-1 / \mathrm{PD}-\mathrm{L} 1$ axis is a promising treatment for TNBCs. However, considering that most BC tumors are not TNBCs, the development of novel effective immunotherapy regardless of subtype and identification of new biomarkers are required.

The classification based on TILs levels and PD-L1 status is considered useful for identifying immune phenotypes of tumors and to indicate the need for immunotherapy (11). TILs indicate the presence of host immune reactions against tumors. PD-L1 can be upregulated in immune cells (ICs) and TCs by inflammatory cytokines including interferon (IFN)- $\gamma$ produced by TILs. Therefore, $\mathrm{TIL}^{+} \mathrm{PD}-\mathrm{L1}^{+}$tumors indicate the presence of adaptive immune resistance, and the blockade of PD-1/PD-L1 interaction by ICIs is an effective strategy for immunotherapy. However, TIL-PD-L1' tumors indicate immune ignorance, implying the occurrence of fewer antitumor responses. A recent clinical trial showed that the benefit of atezolizumab was significantly associated with the high proportion of TILs with upregulation of PD-L1 in ICs from metastatic TN patients $(9,12)$, supporting that this concept can be useful. However, this classification has not been fully applied to BC.

Both antitumor and tumorigenic responses simultaneously occur in the tumor microenvironment (TME). For example, $\mathrm{CD}^{+} \mathrm{T}$ cells prevent tumor progression by eliminating immunogenic TCs. In contrast, $\mathrm{CD}^{+} \mathrm{T}$ helper $2\left(\mathrm{~T}_{\mathrm{H}} 2\right)$ cells and forkhead box (FOX) $\mathrm{P}^{+} \mathrm{T}$ regulatory cells (Tregs) contribute to tumor progression and immunosuppression. Notably, tumor-associated macrophages (TAMs), differentiated from circulating monocytes, enhance tumor progression and distant metastasis by promoting TC invasion, migration, and angiogenesis (13). In particular, TAMs potentially suppress the recruitment of $\mathrm{T}$ cells which attack tumor cells and upregulate immune checkpoint proteins including PD-L1 and regulate the secretion of inhibitory cytokines including TGF- $\beta$ or IL-10 (14). Furthermore, TAMs reportedly inhibit the efficacy of ICIs through several mechanisms (15-17). Macrophages are often investigated within the binary M1 (anti-tumorigenic)-M2 (pro-tumorigenic) polarization system. While CD68 is frequently used as a pan-macrophage marker for both M1 and M2 macrophages (18), CD204, a class A scavenger receptor, has been used as a novel M2-macrophage marker. A high density of CD204 $4^{+}$macrophages is associated with poor prognosis in several cancer types (19-21). These data suggest that TAMs play critical roles to modulate cancer progression. Here, the question remains what the proportion of suppressive subsets, such as TAMs and $\mathrm{FOXP}^{+}$Tregs, are present in BCs, particularly in tumors with high PD-L1 expression and high levels of TILs.

The aims of this study were as follows: i) To classify breast tumors into four groups based on the level of TILs and PD-L1 status regardless of subtype; and ii) to identify the presence of suppressive immune subsets, including TAMs and FOXP3 ${ }^{+}$ Tregs, in primary BCs to assess potential novel immunotherapeutic targets for breast cancer.
Table I. Characteristics of patients $(n=73)$.

\begin{tabular}{|c|c|}
\hline Characteristics & Value \\
\hline Median age, years (range) & $57(26-82)$ \\
\hline T factor, $\mathrm{n}(\mathrm{T} 1 / \mathrm{T} 2 / \mathrm{T} 3 / \mathrm{T} 4)$ & $39 / 32 / 1 / 1$ \\
\hline $\mathrm{N}$ factor, $\mathrm{n}\left(\mathrm{N}^{-} / \mathrm{N}^{+}\right)$ & $42 / 31$ \\
\hline Clinical stage (I/II/III) & 29/35/9 \\
\hline Subtype, n $\left(\mathrm{HR}^{+} \mathrm{HER} 2^{-} / \mathrm{HR}^{+} \mathrm{HER} 2^{+} /\right.$ & $45 / 6 / 3 / 19$ \\
\hline HR-HER2 $\left.^{+} / \mathrm{TN}\right)$ & \\
\hline Histological grade, $n(1 / 2 / 3)$ & $25 / 23 / 25$ \\
\hline Ki-67 index, $\mathrm{n}(\leq 20 />20 \%)$ & $30 / 43$ \\
\hline Histology, $\mathrm{n}$ (invasive ductal carcinoma/others ${ }^{\mathrm{a}}$ ) & $63 / 10$ \\
\hline
\end{tabular}

a4 lobular carcinoma, 3 mucinous carcinoma, 2 apocrine carcinoma and 1 metaplastic carcinoma. HR, hormone receptor; TN, triple-negative.

\section{Patients and methods}

Patients. Seventy-three patients with invasive BC, who underwent surgery for stage I to III tumors from January to November 2017 at Mie University Hospital (Tsu, Japan), were included in this study. Patients with ductal carcinomas in situ (DCIS), de novo stage IV tumors, tumors who received neoadjuvant chemotherapy, and had recurrent tumors were excluded. Table I shows the clinical and pathological characteristics of the patients enrolled in this study.

This study was conducted in accordance with ethical principles, including the Helsinki declaration, and was approved by the Institutional Review Board of Mie University Hospital (No. 3155). The requirement for written informed consent was waived owing to the retrospective nature of the study.

Histological evaluation. All patients received surgical intervention for primary breast tumors. All specimens were formalin-fixed, paraffin-embedded, and cut into 4- $\mu \mathrm{m}$-thick sections for hematoxylin and eosin (H\&E) staining. Histological grades (HGs) were assigned based on the Nottingham system (22), using surgical specimens.

TILs evaluation. H\&E-stained slides were reviewed by two pathologists in accordance with the criteria of the International TILs Working Group 2014 (23). Briefly, lymphoplasmacytic infiltration was evaluated in the stromal area around the invasive tumor, and the average of several tumor areas was determined, excluding the lymphocyte infiltration around DCIS and normal lobules (Fig. 1A and B). TILs were analyzed as a continuous parameter and categorized into two groups using $10 \%$ as a cutoff value, where $<10 \%$ stromal TILs were defined as the low TILs group, and $>10 \%$ was the high TILs group.

Immunohistochemistry (IHC) analysis. Estrogen receptor (ER) and progesterone receptor (PgR) positivity were evaluated against a cutoff of $1 \%$, and HER2 overexpression was evaluated in accordance with the American Society of Clinical Oncology/College of American Pathologists 2013 guidelines. Fluorescence in situ hybridization for assessing HER2 


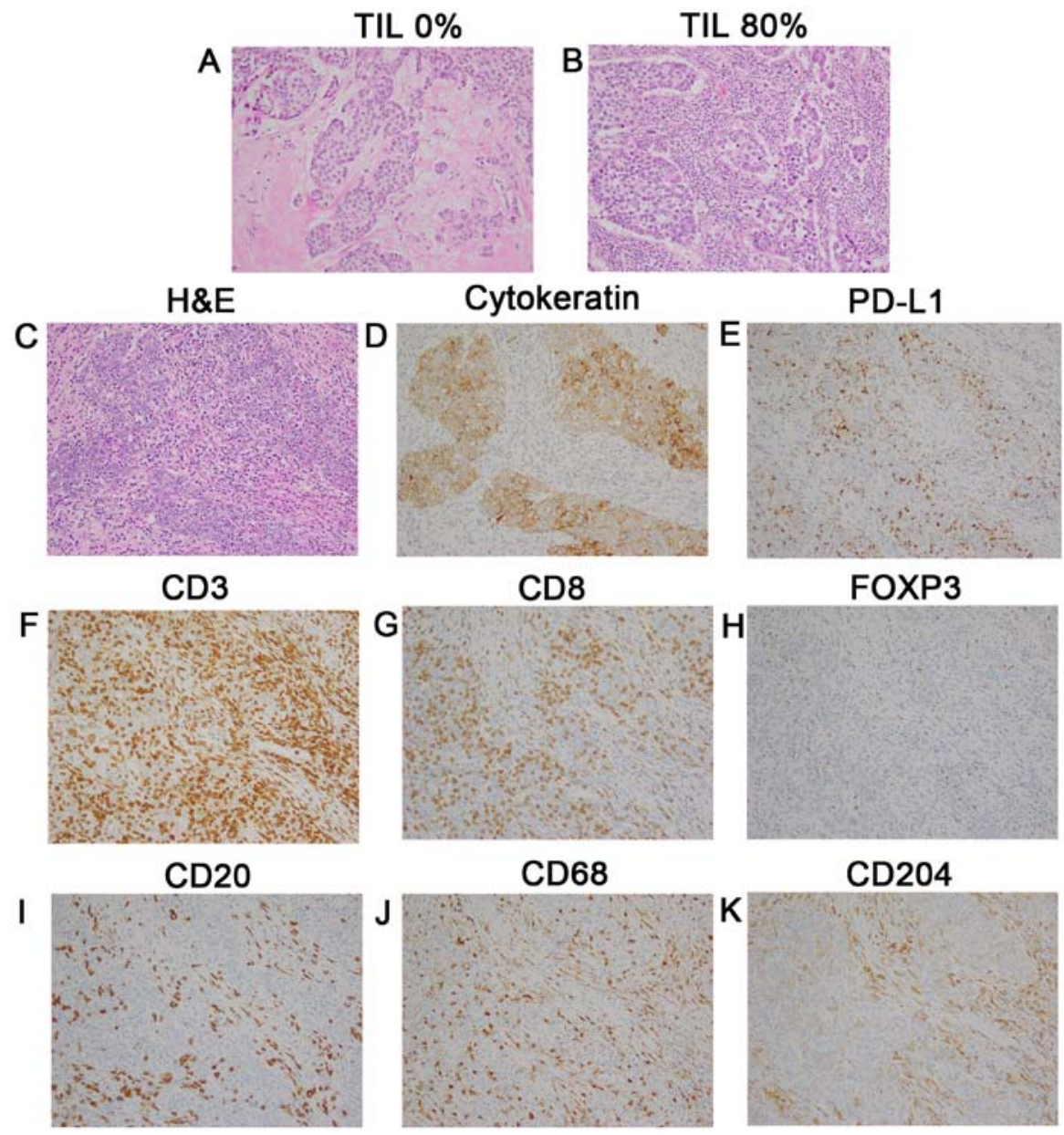

Figure 1. Representative images of TILs and immune subsets evaluated by IHC staining. Magnification, x200. (A) TILs, $0 \%$; and (B) TILs, $80 \%$. TMA samples of a triple-negative tumor (stage II; HG 3; Ki-67 80\%) according to (C) H\&E staining, (D) cytokeratin, (E) PD-L1 expression (3+ on TCs and 2+ on ICs), (F) $\mathrm{CD}^{+},(\mathrm{G}) \mathrm{CD}^{+}$, (H) $\mathrm{FOXP3}^{+}$, (I) $\mathrm{CD} 20^{+}$, (J) $\mathrm{CD}^{2} 8^{+}$macrophages, (K) CD204 $4^{+}$macrophages. TILs, tumor-infiltrating lymphocytes; IHC, immunohistochemical; TCs, tumor cells; ICs, immune cells.

amplification was performed whenever equivocal results $(2+)$ were rendered. Ki-67 expression was assessed via IHC, using the MIB-1 monoclonal antibody (Table SI). In the present study, the cutoff of Ki-67 was $20 \%$, which was the median value in our institute.

IHC was performed using tissue microarrays (TMAs). TMAs were constructed from tumor blocks of surgical specimens, using 2.0-mm (diameter) tumor cores from selected blocks. These cores were assembled in a TMA format, and the paraffin-embedded TMA blocks were then sectioned at 4- $\mu \mathrm{m}$ thickness and subjected to IHC analysis.

Primary antibodies targeting $\mathrm{CD}^{+}, \mathrm{CD}^{+}, \mathrm{FOXP}^{+}$, $\mathrm{CD} 20^{+}, \mathrm{CD}^{+} 8^{+}$, and CD204 ${ }^{+}$cells for IHC and the IHC procedures are described in the supplementary data (Table SI). All IHC staining was performed using an automatic immunostainer (BenchMark XT; Ventana Medical Systems, Tucson, AZ, USA).

Evaluation of PD-L1 expression. The PD-L1 was stained using SP142 (Table SI). The PD-L1 status was evaluated based on the tumor area proportion occupied by PD-L1-expressing tumor-infiltrating immune cells (\% IC) at any intensity or the percentage of PD-L1-expressing tumor cells (\%TC) at any intensity (Fig. 1C-E). According to the result of the clinical trial that used SP142 for TNBCs (10), PD-L1 expression was assessed against a cutoff of $1 \%$.

Evaluation of immune cells. Stained slides were digitized by a slide scanner at a magnification of x200 and 2-3 independent areas with the highest abundance of ICs. All stained cells at both the stroma and tumor nests, regardless of intensity, were evaluated using ImageJ software (National Institutes of Health). Tumors were divided into high or low groups depending on the mean value of the stained area in each subset.

Statistical analysis. Continuous data were analyzed using the Mann-Whitney U or Kruskal-Wallis test followed by Dunn's multiple comparison test. Non-continuous data were compared using Fisher's exact test (two-sided), and $\mathrm{P}<0.05$ indicated statistically significant differences. Correlations between the TIL frequency and the Ki-67 index were evaluated using the Pearson correlation coefficient. Statistical analyses were performed using Prism 7 software (GraphPad Software, Inc.).

\section{Results}

Distribution of TILs in primary BCs. First, we examined the frequency of TILs in 73 primary breast tumors, of which $12 \%$ 


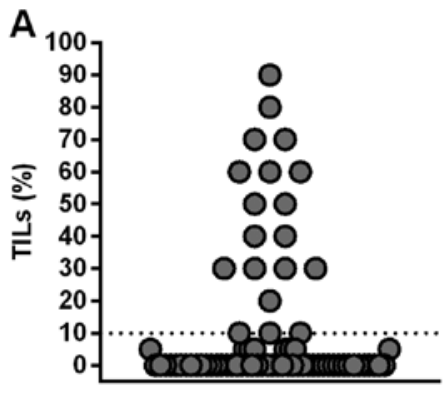

All samples

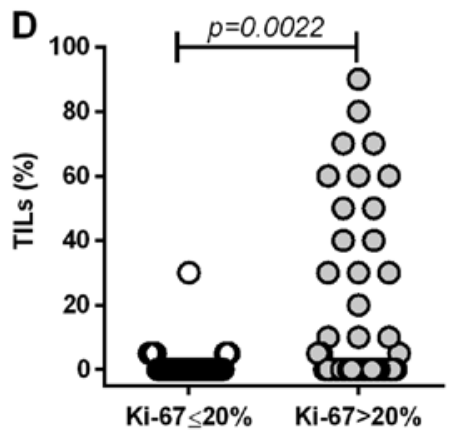

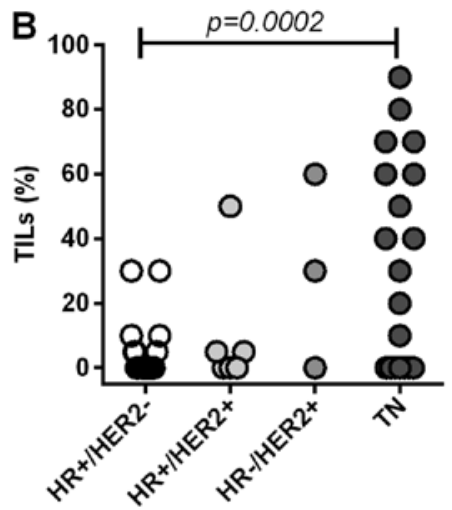

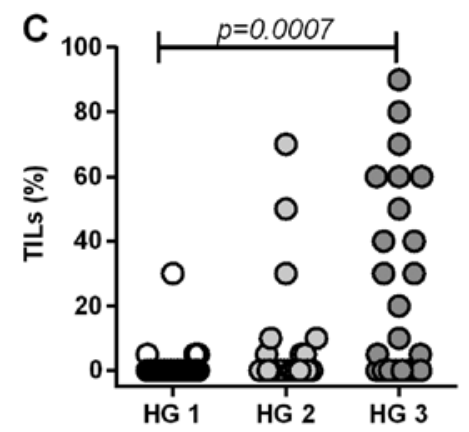

Figure 2. Distribution of TILs based on clinicopathological parameters. (A) All samples (n=73), (B) according to subtypes, (C) HG1-3, (D) Ki-67 index $(\leq 20$ and $>20 \%)$ and (E) correlation between Ki-67 index and TIL distribution. Statistical analyses involved the (B and C) Kruskal-Wallis test and (D) Mann-Whitney U test. (A) The dotted line represents a cutoff value (10\%) of TILs. TILs, tumor-infiltrating lymphocytes; HG, histological grade; $\mathrm{HR}$, hormone receptor; $\mathrm{TN}$, triple-negative.

(9/73) harbored $>50 \%$ TILs, $14 \%(10 / 73)$ harbored $10 \%$ to $50 \%$ TILs, and $74 \%$ (54/73) harbored $<10 \%$ TILs (Fig. 2A). TILs were significantly more abundant in TN tumors than in $\mathrm{HR}^{+}$HER2- tumors $(\mathrm{P}=0.0002)$ (Fig. 2B). Furthermore, TIL levels were significantly higher in tumors with a high $\mathrm{HG}$ than in tumors with a low HG (HG3 vs. HG1, P=0.0007) (Fig. 2C), and in more proliferative tumors $(\mathrm{Ki}-67>20 \%)$ than in less proliferative tumors (Ki-67 $\leq 20 \%)(\mathrm{P}=0.0022)$ (Fig. 2D). TILs levels significantly correlated with the Ki-67 index $(\mathrm{P}<0.0001)$ (Fig. 2E). These results suggest that the frequency of TILs is highly associated with aggressive features of primary tumors. Our results are concurrent with previous findings $(6,24)$. For further analysis, we categorized tumors into two groups (high or low) in accordance with the frequency of TILs (10\% cutoff) (Fig. 2A).

PD-L1 expression on TCs and ICs in primary BCs. We analyzed PD-L1 expression in both TCs and ICs (Table II). For all subtypes, $16.4 \%(12 / 73)$ of tumors were positive for PD-L1 in TCs, while $30.1 \%(22 / 73)$ were positive in ICs. Among $\mathrm{HR}^{+} \mathrm{HER} 2^{-}$tumors, 6.7\% (3/45) and 15.6\% (7/45) were positive for PD-L1 in TCs and ICs, respectively. Among $\mathrm{HR}^{+} \mathrm{HER} 2^{+}$tumors, $0 \%(0 / 6)$ and $33.3 \%(2 / 6)$ were positive for PD-L1 in TCs and ICs, respectively. Among HR-HER2 ${ }^{+}$ tumors, $33.3 \%(1 / 3)$ and $66.7 \%$ (2/3) tumors were positive for PD-L1 in TCs and ICs, respectively. In contrast, among TN tumors, $42.1 \%(8 / 19)$ and $57.9 \%$ (11/19) of tumors were positive for PD-L1 in TCs and ICs, respectively. PD-L1 expression levels tended to be higher in ICs than in TCs, and higher in the TN type than in the HR+HER2- type, concurrent with previous reports (25). Furthermore, PD-L1 expression in both TCs and ICs was significantly associated with high HG (HG 3 vs. HG1-2) (TC: $\mathrm{P}=0.0002$; IC: $\mathrm{P}=0.0001$ ), high Ki-67 index (Ki-67 >20\%) (TC: $\mathrm{P}=0.0215$; IC: $\mathrm{P}=0.0037)$, and high frequency of TILs (TC: $\mathrm{P}<0.0001$; IC: $\mathrm{P}<0.0001)$ (Table III).

Classification of tumors based on TILs and PD-L1 status in primary BCs. Based on the TILs and PD-L1 status, we categorized 73 primary BCs into four groups (Table IV). As shown in Table II, we considered the PD-L1 status in ICs as the entire PD-L1 status in tumor sites. Among all tumors examined, $21.9 \%(16 / 73)$ of tumors were classified as $\mathrm{TIL}^{+} \mathrm{PD}-\mathrm{L}^{+}$, and $65.7 \%$ (48/73) were classified as TIL-PD-L1 ${ }^{-}$. TIL+PD-L1 ${ }^{-}$ group and TIL-PD-L1 ${ }^{+}$group was $4.1 \%(3 / 73)$ and $8.3 \%$ (6/73), respectively. Among TIL+PD-L1+ ${ }^{+}$group, $18.7 \%$ (3/16), $0 \%(0 / 16), 12.5 \%(2 / 16)$, and $68.7 \%(11 / 16)$ were HR+HER2 ${ }^{-}$, $\mathrm{HR}^{+} \mathrm{HER} 2^{+}, \mathrm{HR}^{-} \mathrm{HER}^{+}$and $\mathrm{TN}$ type, respectively. In contrast, among TIL-PD-L1' ${ }^{-}$group, $77.1 \%$ (37/48), $6.3 \%$ (3/48), $2.0 \%$ (1/48), and $14.6 \%$ (7/48) were HR+HER2-, HR ${ }^{+}$HER2 ${ }^{+}$, $\mathrm{HR}^{-} \mathrm{HER}^{+}{ }^{+}$and TN types, respectively. Conversely, $82.2 \%$ (37/45) of HR+HER2- tumors were TIL-PD-L1', while $57.9 \%$ $(11 / 19)$ of TN tumors were TIL + PD-L1 ${ }^{+}$tumors.

Lymphocyte composition in breast cancer tissues. We identified lymphocytes $\left(\mathrm{CD}^{+}, \mathrm{CD}^{+} \mathrm{T}\right.$ cells, FOXP3 ${ }^{+}$Tregs and $\mathrm{CD} 20^{+} \mathrm{B}$ cells) and macrophages $\left(\mathrm{CD}^{+} 8^{+}\right.$and $\mathrm{CD} 204^{+}$cells) in tumor tissues via IHC (Fig. 1F-K) to examine local immune TME. Stained-cell areas were counted using ImageJ software 
Table II. PD-L1 expression in TCs and ICs.

\begin{tabular}{|c|c|c|c|c|c|}
\hline \multirow[b]{2}{*}{ Subtype } & \multirow[b]{2}{*}{ No. } & \multicolumn{2}{|c|}{ PD-L1 expression in TCs } & \multicolumn{2}{|c|}{ PD-L1 expression in ICs } \\
\hline & & Negative, n (\%) & Positive $^{\mathrm{a}}, \mathrm{n}(\%)$ & Negative, n (\%) & Positive $^{\mathrm{a}}, \mathrm{n}(\%)$ \\
\hline $\mathrm{HR}^{+} \mathrm{HER} 2^{-}$ & 45 & $42(93.3)$ & $3(6.7)$ & $38(84.4)$ & $7(15.6)$ \\
\hline $\mathrm{HR}^{+} \mathrm{HER} 2^{+}$ & 6 & $6(100.0)$ & $0(0)$ & $4(66.7)$ & $2(33.3)$ \\
\hline HR-HER2 $^{+}$ & 3 & $2(66.7)$ & $1(33.3)$ & $1(33.3)$ & $2(66.7)$ \\
\hline $\mathrm{TN}$ & 19 & $11(57.9)$ & $8(42.1)$ & $8(42.1)$ & $11(57.9)$ \\
\hline All & 73 & $61(83.6)$ & $12(16.4)$ & $51(69.9)$ & $22(30.1)$ \\
\hline
\end{tabular}

${ }^{\text {aPD}}$-L1 expression was evaluated using SP142 antibody, and judged using a 1\% cutoff. TCs, tumor cells; ICs, immune cells; HR, hormone receptor; HER2, human epidermal growth factor receptor 2; TN, triple-negative.

Table III. Association between PD-L1 expression in TCs/ICs and clinicopathological parameters.

\begin{tabular}{|c|c|c|c|c|c|c|c|}
\hline \multirow[b]{2}{*}{ Variables } & \multirow[b]{2}{*}{ No. } & \multicolumn{3}{|c|}{ PD-L1 in TCs } & \multicolumn{3}{|c|}{ PD-L1 in ICs } \\
\hline & & Negative, $\mathrm{n}$ & Positive, $\mathrm{n}^{\mathrm{a}}$ & P-value ${ }^{b}$ & Negative, $\mathrm{n}$ & Positive, $\mathrm{n}$ & $\mathrm{P}$-value ${ }^{\mathrm{b}}$ \\
\hline \multicolumn{8}{|l|}{$\mathrm{HG}$} \\
\hline $1-2$ & 48 & 46 & 2 & 0.0002 & 41 & 7 & 0.0001 \\
\hline 3 & 25 & 15 & 10 & & 10 & 15 & \\
\hline \multicolumn{8}{|l|}{$\mathrm{Ki}-67, \%$} \\
\hline$\leq 20$ & 30 & 29 & 1 & 0.0218 & 26 & 3 & 0.0037 \\
\hline$>20$ & 43 & 33 & 10 & & 25 & 19 & \\
\hline \multicolumn{8}{|l|}{ TILs, $\%$} \\
\hline$<10$ & 54 & 51 & 2 & $<0.0001$ & 48 & 6 & $<0.0001$ \\
\hline$\geq 10$ & 19 & 10 & 10 & & 3 & 16 & \\
\hline
\end{tabular}

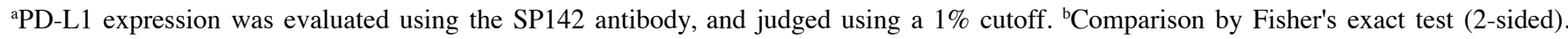
HG, histological grade; TILs, tumor-infiltrating lymphocytes; TCs, tumor cells; ICs, immune cells.

Table IV. Classification of primary breast cancer based on TIL levels and PD-L1 status.

\begin{tabular}{|c|c|c|c|c|}
\hline \multirow[b]{2}{*}{ Subtype } & \multicolumn{4}{|c|}{ Classification of BCs, n (\%) } \\
\hline & $\mathrm{TIL}^{+\mathrm{a} P D-L 1^{+b}}$ & TIL-PD-L1- & $\mathrm{TIL}^{+} \mathrm{PD}-\mathrm{L} 1^{-}$ & TIL-PD-L1 ${ }^{+}$ \\
\hline $\mathrm{HR}^{+} \mathrm{HER}^{-}$ & $3(18.7)$ & $37(77.1)$ & $1(33.3)$ & $4(66.7)$ \\
\hline $\mathrm{HR}^{+} \mathrm{HER}^{+}{ }^{+}$ & $0(0)$ & $3(6.3)$ & $1(33.3)$ & $2(33.3)$ \\
\hline HR $^{-}{ }^{-H E R} 2^{+}$ & $2(12.5)$ & $1(2.0)$ & $0(0)$ & $0(0)$ \\
\hline $\mathrm{TN}$ & $11(68.8)$ & 7 (14.6) & $1(33.3)$ & $0(0)$ \\
\hline Total & $16(21.9)$ & $48(65.7)$ & $3(4.1)$ & $6(8.3)$ \\
\hline
\end{tabular}

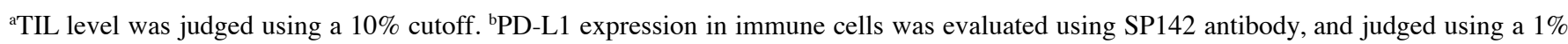
cutoff. HR, hormone receptor; HER2, human epidermal growth factor receptor 2; TIL, tumor-infiltrating lymphocyte; TN, triple-negative.

and the distribution was evaluated for each subset (Fig. 3A). As shown in Fig. $3 \mathrm{~A}, \mathrm{CD}^{+} \mathrm{T}$ cells (total $\mathrm{T}$ cells) were highly accumulated (mean: $6.4 \%$ ) with nearly half of the $\mathrm{T}$ cells determined to be $\mathrm{CD}^{+} \mathrm{T}$ cells (mean: $3.1 \%$ ). $\mathrm{CD} 20^{+} \mathrm{B}$ cells were also accumulated in certain tumors (mean: $2.8 \%$ ). Since we identified FOXP3 ${ }^{+}$Tregs through nuclear staining, the positive area seemed smaller (mean: 0.4) than that in other cells evaluated through surface staining. Nevertheless, we observed the presence of FOXP $3^{+}$cells in some tumors by this method (Fig. 1H). We categorized tumors into a 'high' or 'low' group based on the mean value of each subset. PD-L1 negativity in ICs and TCs was significantly associated with a 

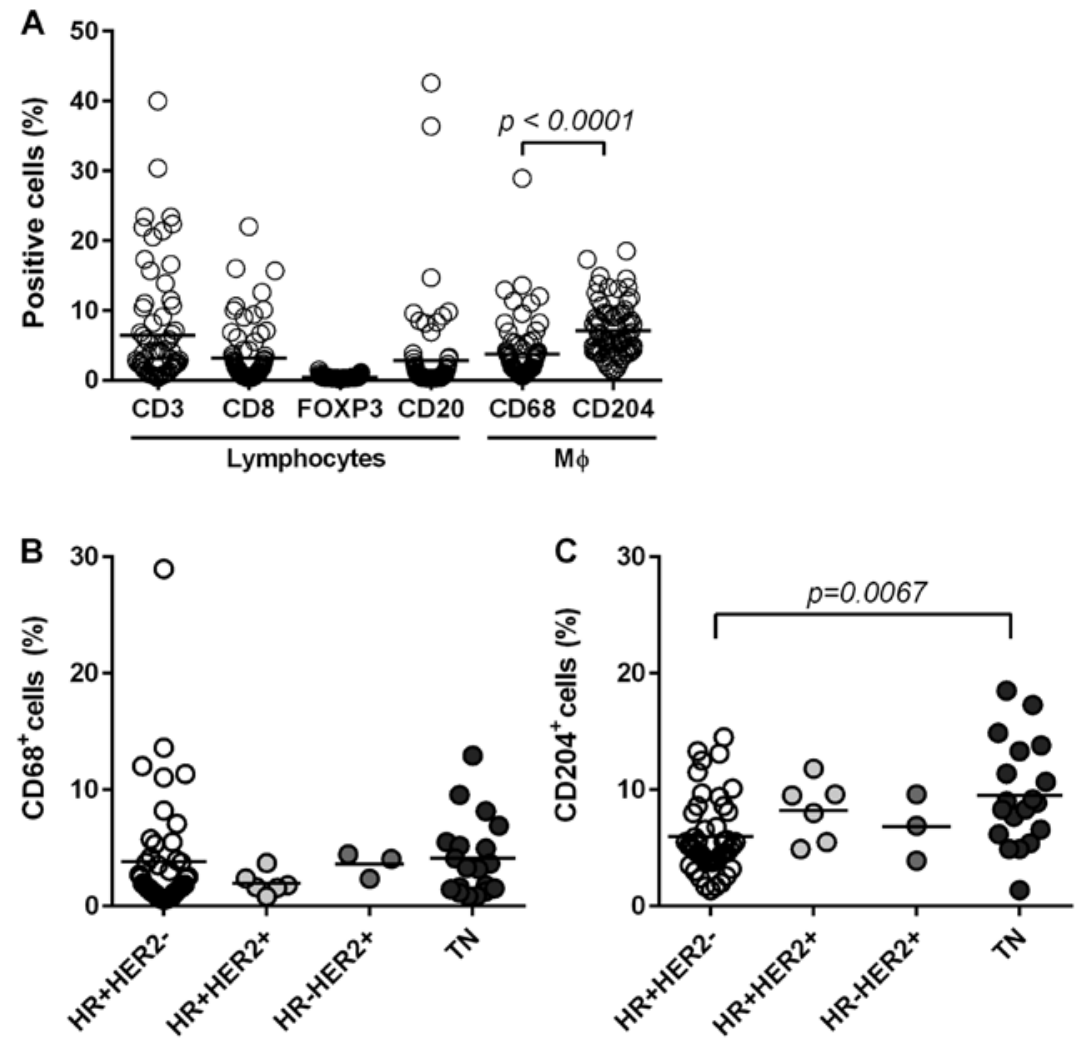

Figure 3. Distribution of immune subsets. (A) Stained slides were digitalized and positive areas were counted using ImageJ software. The distribution of each subset ( $\mathrm{n}=73)$ is shown. The distributions of (B) CD68 ${ }^{+}$macrophages, and (C) CD204 $4^{+}$macrophages are shown according to subtypes. Statistical analyses involved the Mann-Whitney U in (A) and the Kruskal-Wallis test in (C). Bars represent the mean value of each subset. HR, hormone receptor; $\mathrm{TN}$, triple-negative.

Table V. Association between PD-L1 expression in TCs/ICs and immune subsets.

\begin{tabular}{|c|c|c|c|c|c|c|c|}
\hline \multirow[b]{2}{*}{ Variables } & \multirow[b]{2}{*}{ No. } & \multicolumn{3}{|c|}{ PD-L1 in TCs } & \multicolumn{3}{|c|}{ PD-L1 in ICs } \\
\hline & & Negative, $\mathrm{n}$ & Positive $^{\mathrm{a}}, \mathrm{n}$ & P-value ${ }^{b}$ & Negative, $\mathrm{n}$ & Positive $^{\mathrm{a}}, \mathrm{n}$ & P-value \\
\hline CD3 & & & & 0.0006 & & & $<0.0001$ \\
\hline Low $^{c}$ & 51 & 48 & 3 & & 46 & 6 & \\
\hline High & 22 & 13 & 9 & & 6 & 16 & \\
\hline CD8 & & & & 0.0014 & & & $<0.0001$ \\
\hline Low & 54 & 50 & 4 & & 46 & 9 & \\
\hline High & 19 & 11 & 8 & & 6 & 13 & \\
\hline FOXP3 & & & & 0.0419 & & & 0.0317 \\
\hline Low & 50 & 45 & 5 & & 39 & 11 & \\
\hline High & 23 & 16 & 7 & & 12 & 11 & \\
\hline CD20 & & & & $<0.0001$ & & & $<0.0001$ \\
\hline Low & 59 & 55 & 4 & & 48 & 11 & \\
\hline High & 14 & 6 & 8 & & 3 & 11 & \\
\hline CD68 & & & & 0.5132 & & & 0.0145 \\
\hline Low & 49 & 42 & 7 & & 39 & 10 & \\
\hline High & 24 & 19 & 5 & & 12 & 12 & \\
\hline CD204 & & & & 0.0024 & & & $<0.0001$ \\
\hline Low & 43 & 41 & 2 & & 39 & 4 & \\
\hline High & 31 & 20 & 10 & & 12 & 18 & \\
\hline
\end{tabular}

${ }^{\text {aPD-L1 }}$ expression was evaluated using the SP142 antibody, and judged using a 1\% cutoff. ${ }^{\mathrm{b} C o m p a r i s o n}$ by Fisher's exact test (2-sided). 'Tumors were categorized into a high or low group based on the mean value of each immune subset. TCs, tumor cells; ICs, immune cells. 
Table VI. Association between CD68 or CD204-positive macrophages and clinicopathological parameters or immune subsets.

\begin{tabular}{|c|c|c|c|c|c|c|c|}
\hline \multirow[b]{2}{*}{ Variables } & \multirow[b]{2}{*}{ No. } & \multicolumn{3}{|c|}{ CD68-positive cells } & \multicolumn{3}{|c|}{ CD204-positive cells } \\
\hline & & Low $^{\mathrm{a}}, \mathrm{n}$ & $\operatorname{High}^{\mathrm{a}}, \mathrm{n}$ & P-value & Low $^{\mathrm{a}}, \mathrm{n}$ & $\operatorname{High}^{\mathrm{a}}, \mathrm{n}$ & $\mathrm{P}$-value ${ }^{\mathrm{b}}$ \\
\hline $\mathrm{HG}$ & & & & 0.6055 & & & 0.0246 \\
\hline $1-2$ & 48 & 31 & 17 & & 33 & 15 & \\
\hline 3 & 25 & 18 & 7 & & 10 & 15 & \\
\hline $\mathrm{Ki}-67, \%$ & & & & 0.8012 & & & 0.0152 \\
\hline$\leq 20$ & 29 & 21 & 9 & & 23 & 7 & \\
\hline$>20$ & 44 & 28 & 15 & & 20 & 23 & \\
\hline TILs, \% & & & & 0.0472 & & & $<0.0001$ \\
\hline$<10$ & 54 & 40 & 14 & & 40 & 14 & \\
\hline$\geq 10$ & 19 & 9 & 10 & & 3 & 16 & \\
\hline CD3 & & & & 0.0004 & & & $<0.0001$ \\
\hline Low & 51 & 41 & 10 & & 38 & 13 & \\
\hline High & 22 & 8 & 14 & & 5 & 17 & \\
\hline CD8 & & & & $<0.0001$ & & & $<0.0001$ \\
\hline Low & 54 & 44 & 10 & & 40 & 14 & \\
\hline High & 19 & 5 & 14 & & 3 & 16 & \\
\hline FOXP3 & & & & 0.0001 & & & 0.0796 \\
\hline Low & 50 & 41 & 9 & & 33 & 17 & \\
\hline High & 23 & 8 & 15 & & 10 & 13 & \\
\hline CD20 & & & & 0.0547 & & & 0.0706 \\
\hline Low & 59 & 43 & 16 & & 38 & 21 & \\
\hline High & 14 & 6 & 8 & & 5 & 9 & \\
\hline
\end{tabular}

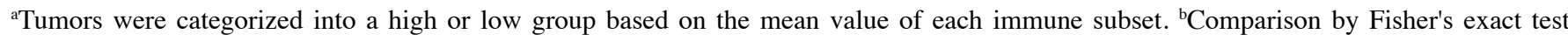
(2-sided). HG, histological grade; TILs, tumor-infiltrating lymphocytes.

low number of $\mathrm{CD}^{+} \mathrm{T}$ cells, $\mathrm{CD} 8^{+} \mathrm{T}$ cells, $\mathrm{CD} 20^{+} \mathrm{B}$ cells and FOXP3 $^{+}$Tregs (Table V).

Macrophage composition in breast cancer tissue and its association with clinicopathological factors. Next, we stained macrophages with two different markers to clarify the characteristics of TAMs in BC tissues: CD68 (a marker of pan-macrophages) and CD204 (a marker of M2-type macrophages) (Fig. 1J and K). Both macrophages were present in the tumor stroma and nest. CD204 ${ }^{+}$macrophages occupied significantly larger areas (mean, 7.1\%) compared to CD68 ${ }^{+}$ macrophages (mean, 3.7\%) in $\mathrm{BC}$ tissues ( $\mathrm{P}<0.0001$; Fig. 3A). Both types of macrophages were associated with high levels of TILs, $\mathrm{CD}^{+} \mathrm{T}$ cells, and $\mathrm{CD}^{+} \mathrm{T}$ cells, but not $\mathrm{CD} 20^{+} \mathrm{B}$ cells (Table VI).

Furthermore, CD204+ M2-type macrophages were significantly higher in TN type compared to in $\mathrm{HR}^{+} \mathrm{HER} 2^{-}$type $(\mathrm{P}=0.0067)$, whereas the frequency of $\mathrm{CD} 68^{+}$macrophages was the same among all subtypes (Fig. 3B and C). Furthermore, high levels of CD204 ${ }^{+}$macrophages were significantly associated with high $\mathrm{HG}(\mathrm{P}=0.0246)$, and high Ki-67 $(\mathrm{P}=0.0152)$. In contrast, $\mathrm{CD}^{+} 8^{+}$macrophages were not associated with these factors (Table VI). Interestingly, CD204+ M2-macrophages significantly accumulated in tumors with PD-L1 upregulation in both TCs and ICs (Table V).
Suppressive subsets in $T I L^{+} P D-L 1^{+}$and TIL-PD-L1- tumors. Finally, we quantified the suppressive cells, FOXP3 ${ }^{+}$Tregs, and CD204 $4^{+}$M2-type macrophages in each of the four groups categorized by TILs and PD-L1 status. The frequency of tumors with high FOXP3 ${ }^{+}$Tregs, and high CD204 ${ }^{+}$macrophages was $62.5 \%(10 / 16)$ and $87.5 \%(14 / 16)$ among TIL+PD-L1+ tumors, respectively (Table VII). By contrast, among TIL-PD-L1tumors, the frequency of tumors with high FOXP ${ }^{+}$Tregs and high $\mathrm{CD} 204^{+}$macrophages were $27.1 \%$ (13/48) and $20.8 \%$ (10/48), respectively. These results indicate that TIL+PD-L1+ tumors, considered responsible for ICIs, contain abundant suppressive cells as infiltrating cell subsets.

\section{Discussion}

In this study, we categorized 73 primary breast tumors into four different groups based on TILs and PD-L1 status. Consequently, $22 \%$ of the tumors were classified as TIL ${ }^{+} \mathrm{PD}-\mathrm{L}^{+}$ and $66 \%$ were as TIL-PD-L1 ${ }^{-}$. Furthermore, suppressive subsets, CD204 ${ }^{+} \mathrm{M} 2$-type macrophages and FOXP3 ${ }^{+}$Tregs were highly accumulated in $\mathrm{TIL}^{+} \mathrm{PD}-\mathrm{L1}^{+}$tumors.

As expected, the $\mathrm{TIL}^{+} \mathrm{PD}-\mathrm{L1}^{+}$group primarily comprised TN types (69\%) known to be highly immunogenic, whereas the TIL-PD-L1'- group primarily included $\mathrm{HR}^{+} \mathrm{HER} 2^{-}$cells $(77 \%)$, suggesting that the classification of BC tumors based on TILs 
Table VII. Distribution of suppressive subsets in each of the four groups categorized by TIL level and PD-L1 status ( $\mathrm{n}=73$ ).

Classification of BCs, n (\%)

\begin{tabular}{lcccc}
\cline { 2 - 4 } Suppressive subset & TIL $^{\text {+a }}$ PD-L1 $^{\text {+b }}$ & TIL PD-L1 & TIL $^{+}$PD-L1 & TIL $^{-}$PD-L1 $^{+}$ \\
\hline FOXP3-low $^{\text {c }}$ & $6(37.5)$ & $35(72.9)$ & $1(33.3)$ & $5(83.3)$ \\
FOXP3-high $^{d}$ & $10(62.5)$ & $13(27.1)$ & $2(66.7)$ & $1(16.7)$ \\
CD204-low $^{\mathrm{e}}$ & $2(12.5)$ & $38(79.2)$ & $1(33.3)$ & $2(33.3)$ \\
CD204-high $^{\text {f }}$ & $14(87.5)$ & $10(20.8)$ & $2(66.7)$ & $4(66.7)$
\end{tabular}

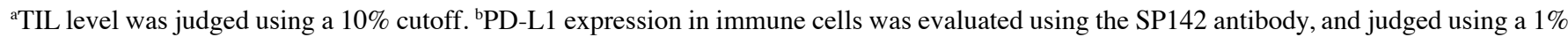
cutoff. 'Tumors with low levels of FOXP3 ${ }^{+}$Tregs. ${ }^{\mathrm{d}}$ Tumors with high levels FOXP3 ${ }^{+}$Tregs. ${ }^{\mathrm{e}}$ Tumors with low levels of CD204 ${ }^{+}$macrophages. ${ }^{\mathrm{f}}$ Tumors with high levels of CD204+ ${ }^{+}$macrophages. TILs, tumor-infiltrating lymphocytes.

and the PD-L1 status mostly corresponded to the classification by subtype. However, these results show that approximately one-third of TIL ${ }^{+} \mathrm{PD}-\mathrm{L1}^{+}$tumors were non-TNBCs and could benefit from immunotherapy, though this population is very small. Conversely, $23 \%$ of TIL-PD-L1' tumors were represented with $\mathrm{HR}^{+} \mathrm{HER} 2^{+}$, HR-HER2 ${ }^{+}$, and TN types.

The molecular classifications of TN tumors have recently been reported. Lehmann et al (26) proposed TN tumors into four stable transcriptional subtypes, basal-like 1 (BL1), basal-like 2 (BL2), mesenchymal (M), and luminal androgen receptor (LAR). Furthermore, Harano et al (27) reported that the rate of immunomodulatory signature was the highest in BL1 $(48 \%)$ and the lowest in M (0\%). We showed that PD-L1 expression was enhanced in aggressive tumors featuring high HG and Ki-67 index with abundant immune cells. Notably, this tendency became more significant in highly proliferative tumors (data not shown). These clinical results may suggest that among TN types, most $\mathrm{TIL}^{+} \mathrm{PD}-\mathrm{L}^{+}{ }^{+}$tumors may have molecular characteristics of basal-like types which show highly proliferative phenotypes. By contrast, some TN tumors categorized as TIL-PD-L1' may have other characteristics such as mesenchymal, or LAR type. In fact, one of the TIL-PD-L1 TN tumor in this study was a metaplastic carcinoma that presented with a mesenchymal characteristic (data not shown). Besides, the sample size was too small to sufficiently assess the association between two types of HER2-positive tumors and PD-L1 expression in this study. A larger study is required to further address this issue.

TIL-PD-L1+ tumors indicate that PD-L1 is upregulated through intrinsic oncogenic induction, such as the effect of the loss of tumor suppression by phosphatase and tensin homolog (PTEN) signals (28). This tumor type is not considered an effective target for ICIs owing to its lack of T cells. Therefore, $\mathrm{T}$ cells should be recruited through other approaches such as chemotherapy and irradiation therapy to induce immune reactions through immunogenic cell death (29).

$\mathrm{TIL}^{+} \mathrm{PD}-\mathrm{L1}^{-}$tumors indicate the presence of immune tolerance by suppressive factors other than adaptive resistance induced by the PD-1/PD-L1 interaction. Lymphocytes in these tumors may become suppressed through several mechanisms such as those involving M2-polarized macrophages, myeloid-derived suppressor cells, or metabolites like indoleamine 2, 3-dioxygenase (IDO) (11).
Herein, we focused on the presence of TAMs to comprehend the local TME. CD68 and CD163 are frequently used markers to identify macrophages in tumor tissue $(18,30)$. In particular, $\mathrm{CD}_{163^{+}}$macrophages are reportedly correlated with a poor prognosis $(18,31)$. Furthermore, recent studies have reported that a high density of $\mathrm{CD} 204^{+}$macrophages is strongly associated with poor prognosis of $\mathrm{BC}$ patients $(19,20)$ as well as in NSCLC (32), gastric cancer (33), and esophageal cancer $(21,34)$. Therefore, we analyzed the presence of $\mathrm{CD}_{20} 4^{+}$macrophages comparing it to $\mathrm{CD} 68^{+}$ macrophages. We observed that $\mathrm{CD} 204^{+}$cells accumulated in more aggressive tumors than did $\mathrm{CD}^{+} 8^{+}$cells in $\mathrm{BC}$ tissue, in line with a previous study (19). Furthermore, we found that $\mathrm{CD} 204^{+}$macrophages were likely to be present in tumors with high PD-L1 expression, indicating that CD204 macrophages may be one of the immune subsets that express PD-L1, thus preventing the function of PD-1 expressing $\mathrm{CD}^{+} \mathrm{T}$ cells. We also found that $\mathrm{TIL}^{+} \mathrm{PD}-\mathrm{L}^{+}{ }^{+} \mathrm{BC}$ tumors, considered responsible for ICIs, included abundant TAMs in tumor tissue, thus potentially accounting for the weak efficacy of ICIs alone for TNBCs $(35,36)$. Currently, clinical trials targeting TAMs, e.g., targeting colony-stimulating factor 1 (CSF1), and chemokine receptor 2 (CCR2), alone or in combination with chemotherapy and/or checkpoint inhibitors are underway (14). Future studies are required to evaluate whether CD204+ cells in BC is an efficacious therapeutic target.

One of the limitations of our study is the small number of cases. In particular, data obtained for HER2-positive tumors were inconclusive owing to the small number of cases enrolled (6 $\mathrm{HR}^{+} \mathrm{HER} 2^{-}$, $3 \mathrm{HR}^{-} \mathrm{HER} 2^{+}$tumors). Furthermore, since cutoff values for immune subsets have not yet been established, we determined high/low values in accordance with our own conditions. A standardized evaluation system is required for immune subsets like TILs (23). Furthermore, we did not determine the prognostic effects of these classifications and high density of CD $204^{+}$macrophages/FOXP $3^{+}$Tregs, given that no data on survival are currently available.

In conclusion, we classified breast tumors into four groups based on TILs and PD-L1 status irrespective of subtype and found that $22 \%$ of tumors were classified as $\mathrm{TIL}^{+} \mathrm{PD}-\mathrm{L1}^{+}$tumors (69\% was TN type), whereas $66 \%$ of tumors were classified as TIL-PD-L1'- tumors (77\% was HR+HER2- type). Furthermore, 
we found that $\mathrm{CD} 204^{+}$macrophages and FOXP3 ${ }^{+}$Tregs were highly accumulated in $\mathrm{TIL}^{+} \mathrm{PD}-\mathrm{L1}^{+}$tumors, which may contribute to immunotherapy resistance. The present results suggest that the evaluation of TILs, PD-L1 expression, and TAMs would help effectively select candidate immunotherapies and the development of novel strategies depending on the immune microenvironment in $\mathrm{BC}$.

\section{Acknowledgements}

Not applicable.

\section{Funding}

No funding was received.

\section{Availability of data and materials}

The datasets used and/or analyzed during the current study are available from the corresponding author on reasonable request.

\section{Authors' contributions}

KS designed the study. MN, KS, AN, NI, MS, MK, MakI, YT, HO, MikI, TM and TO contributed to acquisition of data. YK, MasI and HY contributed to pathological analysis. KS, MN, YK and TM contributed to analysis and interpretation of data. KS and MN drafted the manuscript. TO and NK were involved in analysis and interpretation of data, and revised the manuscript critically for important intellectual content. All authors read and approved the final manuscript.

\section{Ethics approval and consent to participate}

The present study was approved by the Institutional Review Board of Mie University Hospital (approval no. 3155). The requirement for written informed consent was waived due to the retrospective nature of the study.

\section{Patient consent for publication}

Not applicable.

\section{Competing interests}

The authors declare that they have no competing interests.

\section{References}

1. Wolchok JD, Kluger H, Callahan MK, Postow MA, Rizvi NA, Lesokhin AM, Segal NH, Ariyan CE, Gordon RA, Reed K, et al: Nivolumab plus ipilimumab in advanced melanoma. N Engl J Med 369: 122-133, 2013.

2. Powles T, Eder JP, Fine GD, Braiteh FS, Loriot Y, Cruz C, Bellmunt J, Burris HA, Petrylak DP, Teng SL, et al: MPDL3280A (anti-PD-L1) treatment leads to clinical activity in metastatic bladder cancer. Nature 515: 558-562, 2014.

3. Herbst RS, Baas P, Kim DW, Felip E, Pérez-Gracia JL, Han JY, Molina J, Kim JH, Arvis CD, Ahn MJ, et al: Pembrolizumab versus docetaxel for previously treated,PD-L1-positive, advanced non-small-cell lung cancer (KEYNOTE-010): A randomised controlled trial. Lancet 387: 1540-1550, 2016.
4. Alexandrov LB, Nik-Zainal S, Wedge DC, Aparicio SA, Behjati S, Biankin AV, Bignell GR, Bolli N, Borg A, Børresen-Dale AL, et al: Signatures of mutational processes in human cancer. Nature 500: 415-421, 2013.

5. Adams S, Gray RJ, Demaria S, Goldstein L, Perez EA, Shulman LN, Martino S, Wang M, Jones VE, Saphner TJ, et al: Prognostic value of tumor-infiltrating lymphocytes in triple-negative breast cancers from two phase III randomized adjuvant breast cancer trials: ECOG 2197 and ECOG 1199. J Clin Oncol 32: 2959-2966, 2014.

6. Loi S, Sirtaine N, Piette F, Salgado R, Viale G, Van Eenoo F, Rouas G, Francis P, Crown JP, Hitre E, et al: Prognostic and predictive value of tumor-infiltrating lymphocytes in a phase III randomized adjuvant breast cancer trial in node-positive breast cancer comparing the addition of docetaxel to doxorubicin with doxorubicin-based chemotherapy: BIG 02-98. J Clin Oncol 31: 860-867, 2013.

7. Loi S, Michiels S, Salgado R, Sirtaine N, Jose V, Fumagalli D, Kellokumpu-Lehtinen PL, Bono P, Kataja V, Desmedt C, et al: Tumor infiltrating lymphocytes are prognostic in triple negative breast cancer and predictive for trastuzumab benefit in early breast cancer: Results from the FinHER trial. Ann Oncol 25: 1544-1550, 2014

8. Adams S, Loi S, Toppmeyer D, Cescon DW, De Laurentiis M Nanda R, Winer EP, Mukai H, Tamura K, Armstrong A, et al: Pembrolizumab monotherapy for previously untreated, PD-L1-positive, metastatic triple-negative breast cancer: Cohort $\mathrm{B}$ of the phase II KEYNOTE-086 study. Ann Oncol 30: 405-411, 2019.

9. Emens LA, Cruz C, Eder JP, Braiteh F, Chung C, Tolaney SM, Kuter I, Nanda R, Cassier PA, Delord JP, et al: Long-term clinical outcomes and biomarker analyses of atezolizumab therapy for patients with metastatic triple-negative breast cancer: A phase 1 study. JAMA Oncol 5: 74-82, 2019.

10. Schmid P, Adams S, Rugo HS, Schneeweiss A, Barrios CH, Iwata H, Diéras V, Hegg R, Im SA, Shaw Wright G, et al: Atezolizumab and nab-paclitaxel in advanced triple-negative breast cancer. N Engl J Med 379: 2108-2121, 2018.

11. Teng MW, Ngiow SF, Ribas A and Smyth MJ: Classifying cancers based on T-cell Infiltration and PD-L1. Cancer Res 75: 2139-2145, 2015.

12. Emens LA, Loi S, Rugo HS, Schneeweiss A, Diéras V, Iwata H, Barrios CH, Nechaeva M, Molinero L, Duc AN, et al: Abstract GS1-04: IMpassion130: Efficacy in immune biomarker subgroups from the global, randomized, double-blind, placebo-controlled, phase III study of atezolizumab + nab-paclitaxel in patients with treatment-naïve, locally advanced or metastatic triple-negative breast cancer. Cancer Res 79 (4 Suppl): GS1-04, 2019.

13. Noy R and Pollard JW: Tumor-associated macrophages: From mechanisms to therapy. Immunity 41: 49-61, 2014.

14. DeNardo DG and Ruffell B: Macrophages as regulators of tumour immunity and immunotherapy. Nat Rev Immunol 19: 369-382, 2019.

15. Zhu Y, Knolhoff BL, Meyer MA, Nywening TM, West BL, Luo J, Wang-Gillam A, Goedegebuure SP, Linehan DC and DeNardo DG: CSF1/CSF1R blockade reprograms tumorinfiltrating macrophages and improves response to T-cell checkpoint immunotherapy in pancreatic cancer models. Cancer Res 74: 5057-5069, 2014.

16. Arlauckas SP, Garris CS, Kohler RH, Kitaoka M, Cuccarese MF, Yang KS, Miller MA, Carlson JC, Freeman GJ, Anthony RM, et al: In vivo imaging reveals a tumor-associated macrophage-mediated resistance pathway in anti-PD-1 therapy. Sci Transl Med 9: eaal3604, 2017.

17. Lo Russo G, Moro M, Sommariva M, Cancila V, Boeri M, Centonze G, Ferro S, Ganzinelli M, Gasparini P, Huber V, et al: Antibody- $\mathrm{Fc} / \mathrm{FcR}$ interaction on macrophages as a mechanism for hyperprogressive disease in non-small cell lung cancer subsequent to PD-1/PD-L1 blockade. Clin Cancer Res 25: 989-999, 2019.

18. Medrek C, Pontén F, Jirström K and Leandersson K: The presence of tumor associated macrophages in tumor stroma as a prognostic marker for breast cancer patients. BMC Cancer 12: 306, 2012.

19. Miyasato Y, Shiota T, Ohnishi K, Pan C, Yano H, Horlad H, Yamamoto Y, Yamamoto-Ibusuki M, Iwase H, Takeya $M$ and Komohara Y: High density of CD204-positive macrophages predicts worse clinical prognosis in patients with breast cancer. Cancer Sci 108: 1693-1700, 2017. 
20. Shimada H, Hasebe T, Sugiyama M, Shibasaki S, Sugitani I, Ueda S, Gotoh Y, Yasuda M, Arai E, Osaki A and Saeki T: Fibrotic focus: An important parameter for accurate prediction of a high level of tumor-associated macrophage infiltration in invasive ductal carcinoma of the breast. Pathol Int 67: 331-341, 2017.

21. Hatogai K, Kitano S, Fujii S, Kojima T, Daiko H, Nomura S, Yoshino T, Ohtsu A, Takiguchi Y, Doi T and Ochiai A: Comprehensive immunohistochemical analysis of tumor microenvironment immune status in esophageal squamous cell carcinoma. Oncotarget 7: 47252-47264, 2016.

22. Elston CW and Ellis IO: Pathological prognostic factors in breast cancer. I. The value of histological grade in breast cancer: Experience from a large study with long-term follow-up. Histopathology 19: 403-410, 1991.

23. Salgado R, Denkert C, Demaria S, Sirtaine N, Klauschen F, Pruneri G, Wienert S, Van den Eynden G, Baehner FL, Penault-Llorca F, et al: The evaluation of tumor-infiltrating lymphocytes (TILs) in breast cancer: Recommendations by an International TILs working group 2014. Ann Oncol 26: 259-271, 2015.

24. Mohammed ZM, Going JJ, Edwards J, Elsberger B, Doughty JC and McMillan DC: The relationship between components of tumour inflammatory cell infiltrate and clinicopathological factors and survival in patients with primary operable invasive ductal breast cancer. Br J Cancer 107: 864-873, 2012.

25. Miglietta F, Griguolo G, Guarneri V and Dieci MV: Programmed cell death ligand 1 in breast cancer: Technical aspects, prognostic implications, and predictive value. Oncologist 24: e1055-e1069, 2019.

26. Lehmann BD, Jovanović B, Chen X, Estrada MV, Johnson KN Shyr Y, Moses HL, Sanders ME and Pietenpol JA: Refinement of triple-negative breast cancer molecular subtypes: Implications for neoadjuvant chemotherapy selection. PLoS One 11: e0157368, 2016.

27. Harano K, Wang Y, Lim B, Seitz RS, Morris SW, Bailey DB Hout DR, Skelton RL, Ring BZ, Masuda H, et al: Rates of immune cell infiltration in patients with triple-negative breast cancer by molecular subtype. PLoS One 13: e0204513, 2018.

28. Parsa AT, Waldron JS, Panner A, Crane CA, Parney IF, Barry JJ, Cachola KE, Murray JC, Tihan T, Jensen MC, et al: Loss of tumor suppressor PTEN function increases B7-H1 expression and immunoresistance in glioma. Nat Med 13: 84-88, 2007.
29. Kroemer G, Galluzzi L, Kepp O and Zitvogel L: Immunogenic cell death in cancer therapy. Annu Rev Immunol 31: 51-72, 2013.

30. Mahmoud SM, Lee AH, Paish EC, Macmillan RD, Ellis IO and Green AR: Tumour-infiltrating macrophages and clinical outcome in breast cancer. J Clin Pathol 65: 159-163, 2012.

31. Tiainen S, Tumelius R, Rilla K, Hämäläinen K, Tammi M, Tammi R, Kosma VM, Oikari S and Auvinen P: High numbers of macrophages, especially M2-like (CD163-positive), correlate with hyaluronan accumulation and poor outcome in breast cancer. Histopathology 66: 873-883, 2015.

32. Li Z, Maeda D, Yoshida M, Umakoshi M, Nanjo H, Shiraishi K, Saito M, Kohno T, Konno H, Saito H, et al: The intratumoral distribution influences the prognostic impact of CD68- and CD204-positive macrophages in non-small cell lung cancer. Lung Cancer 123: 127-135, 2018.

33. Ichimura $\mathrm{T}$, Abe $\mathrm{H}$, Morikawa $\mathrm{T}$, Yamashita $\mathrm{H}$, Ishikawa $\mathrm{S}$, Ushiku T, Seto Y and Fukayama M: Low density of CD204-positive M2-type tumor-associated macrophages in Epstein-Barr virus-associated gastric cancer: A clinicopathologic study with digital image analysis. Hum Pathol 56: 74-80, 2016.

34. Yagi T, Baba Y, Okadome K, Kiyozumi Y, Hiyoshi Y, Ishimoto T, Iwatsuki M, Miyamoto Y, Yoshida N, Watanabe M, et al: Tumour-associated macrophages are associated with poor prognosis and programmed death ligand 1 expression in oesophageal cancer. Eur J Cancer 111: 38-49, 2019.

35. Nanda R, Chow LQ, Dees EC, Berger R, Gupta S, Geva R, Pusztai L, Pathiraja K, Aktan G, Cheng JD, et al: Pembrolizumab in patients with advanced triple-negative breast cancer: Phase Ib KEYNOTE-012 study. J Clin Oncol 34: 2460-2467, 2016.

36. Adams S, Schmid P, Rugo HS, Winer EP, Loirat D, Awada A, Cescon DW, Iwata H,Campone M, Nanda R, et al: Pembrolizumab monotherapy for previously treated metastatic triple-negative breast cancer: Cohort A of the phase II KEYNOTE-086 study. Ann Oncol 30: 397-404, 2019.

(i)(5) This work is licensed under a Creative Commons Attribution-NonCommercial-NoDerivatives 4.0 International (CC BY-NC-ND 4.0) License. 